\title{
Politique énergétique allemande et agriculture au Jura souabe : denrées agricoles ou méthane?
}

German Energy Policy and Agriculture in Swabian Jura: Agricultural Commodities or Methane?

\section{Sascha Vue et Nadège Garambois}

\section{(2) OpenEdition}

Journals

Édition électronique

URL : http://journals.openedition.org/economierurale/5363

DOI : 10.4000/economierurale.5363

ISSN : 2105-2581

Éditeur

Société Française d'Économie Rurale (SFER)

Édition imprimée

Date de publication : 15 décembre 2017

Pagination : 49-64

ISSN : 0013-0559

Référence électronique

Sascha Vue et Nadège Garambois, «Politique énergétique allemande et agriculture au Jura souabe : denrées agricoles ou méthane? », Économie rurale [En ligne], 362 | Novembre-décembre 2017, mis en ligne le 15 décembre 2019, consulté le 03 janvier 2020. URL : http://journals.openedition.org/ economierurale/5363 ; DOI : 10.4000/economierurale.5363

(c) Tous droits réservés 


\title{
Politique énergétique allemande et agriculture au Jura souabe : denrées agricoles ou méthane?
}

\author{
Sascha VUE • Agro-économiste, Le Bourg, Vabre Tizac 12240 Le Bas Ségala \\ sascha.v@riseup.net \\ Nadège GARAMBOIS • UFR Agriculture comparée et développement agricole/ \\ UMR Prodig, Agroparistech, Paris \\ nadege.garambois@agroparistech.fr
}

\begin{abstract}
Depuis 1990, I'Allemagne s'est progressivement dotée d'une politique ambitieuse de soutien aux énergies renouvelables qui prévoit des prix bonifiés de l'électricité au producteur et s'est traduite dans le Jura souabe, comme dans tout le sud du pays, d'un mouvement rapide d'installations d'unités de méthanisation depuis une dizaine d'années, modifiant ainsi en profondeur cette agriculture de moyenne montagne. La production de méthane est réalisée au détriment de celles de lait et de grains et pénalise in fine l'emploi agricole, tandis que le prix de l'électricité, artificiellement élevé de 2009 à 2012 et garanti sur vingt ans, contribue à accroître les écarts de revenus et les prix du foncier. Cette politique fédérale s'inscrit en complet décalage avec les mesures de politique agricole à l'échelle du Land du Bade-Wurtemberg, qui privilégie emploi agricole et préservation des écosystèmes locaux.
\end{abstract}

MOTS-CLÉS : agriculture, développement durable, politique énergétique allemande, méthaniseur, agriculture comparée

\section{German Energy Policy and Agriculture in Swabian Jura: Agricultural Commodities or Methane?}

Since 1990, Germany has gradually adopted an ambitious policy support for renewable energy that provides subsidized electricity prices to the producer and resulted, in the Swabian Alps as throughout Southern-Germany, in a rapid movement of biogas plants installations for a decade, which fundamentally changes this middle mountain agriculture. Methane is provided at the expense of milk and grains and its production increase penalizes agricultural employment, while the price of electricity artificially high from 2009 to 2012 and secured over twenty years, contributes to increasing income disparities and higher land prices. This federal policy is out of step with the agricultural policy of Baden-Württemberg, which favors agricultural employment and preservation of local ecosystems. (JEL: Q00)

KEYWORDS: agriculture, sustainable development, German energy policy, biogas plant, compared agriculture

$D_{1}$ epuis le début des années 1990, 'Allemagne s'est dotée d'une politique ambitieuse de soutien aux énergies renouvelables (EEG : Erneuebare-EnergienGesetz), qui prévoit des prix bonifiés de l'électricité au producteur. Depuis une vingtaine d'années, les budgets dédiés et les efforts de recherche ont été successivement concentrés sur l'éolien, le solaire, l'éthanol et plus récemment le méthane, en orientant les soutiens afin de privilégier une production assurée en premier lieu par des petites et moyennes unités (Mautz et al., 2008). Pour l'Allemagne, cette politique vise aussi bien à diversifier ses sources d'énergie qu'à développer son industrie et à se 
perfectionner sur le plan technologique. Avec plus du tiers de sa production d'énergie primaire issue d'énergies dites « renouvelables », l'Allemagne est aujourd'hui le premier producteur mondial d'électricité solaire, le second pour sa production d'électricité à partir de la biomasse et le quatrième pour sa production d'électricité éolienne ${ }^{1}$. Grâce à des dispositifs incitatifs, la production de méthane qui représente aujourd'hui en Allemagne plus de $20 \%$ des énergies renouvelables et $7 \%$ de la production d'énergie primaire ${ }^{2}$, a connu un récent et rapide développement outre-Rhin (passée de 1050 MWh en 2000 à plus de $2500 \mathrm{MWh}$ en 2010) (DBEW, 2013), particulièrement dans le sud du pays et tout spécialement au sein des exploitations agricoles.

Cet article s'intéresse aux conséquences de la mise en œuvre de cette politique énergétique sur les dynamiques agraires au sein des régions de moyenne montagne du sud de l'Allemagne, où avec $24 \%$ et $9 \%$ de la production nationale, la Bavière et le Bade-Wurtemberg se placent respectivement en deuxième et troisième position des Länder pour la production d'électricité à partir de méthane (DBFZ, 2014). Cette recherche vise également à apprécier les effets comparés de cette politique énergétique fédérale sur l'agriculture souabe et sa durabilité, au regard des autres outils de politique agricole et rurale existants dans ces régions, pour laquelle la compétence est partagée entre Fédération et Länder (Thoroe, 2002). Afin d'étudier finement ces effets, l'étude porte sur une petite région du Bade-Wurtemberg, le Mittlere Alb ou Jura souabe moyen (canton de Reutlingen), caractéristique du sud de l'Allemagne pour sa combinaison d'énergies renouvelables centrée sur le solaire

1. D'après les statistiques de l'Agence internationale de l'énergie (2015).

2. Ibid. et le méthane (Deshaies, 2014), où prédominent une agriculture familiale et l'élevage bovin laitier. Le Jura souabe connaît depuis une dizaine d'années un mouvement rapide d'installations d'unités de méthanisation au sein d'une partie des exploitations agricoles. Celui-ci bouleverse l'agriculture locale et permet désormais au seul canton de Reutlingen d'assurer près de $10 \%$ de la production de méthane du Bade-Wurtemberg.

Cette recherche s'inscrit dans le programme ANR Bip-Pop (2011-2015) ${ }^{3}$. Elle repose sur un diagnostic agraire (Cochet et Devienne, 2006 ; Cochet et al., 2007) mené dans la région du Jura souabe moyen et basé sur six mois de travail de terrain (Vue, 2012). Sur la base d'un échantillonnage raisonné, trente entretiens historiques et cinquante enquêtes technico-économiques ont été conduits auprès d'agriculteurs de la région. Les enquêtes historiques ont permis de reconstituer les dynamiques agraires du Jura souabe, d'étudier l'impact des politiques agricoles et énergétique sur ces évolutions et de reconstituer la différenciation progressive des systèmes de production permettant ainsi d'établir une typologie des différents systèmes de production actuels dans la région. Un système de production est ici conçu comme un accès aux ressources (localisation et surface, équipement, main-d'œuvre), une combinaison de systèmes de culture et de systèmes d'élevage et une combinaison de productions, communs à plusieurs exploitations de la région (Reboul, 1976). En mettant en œuvre la méthode explicitée par Cochet et al.

3. Le programme ANR Bip-Pop a porté sur l'analyse des effets des politiques publiques, sur la production de biens publics par l'agriculture et plus spécifiquement, dans un volet traitant de l'impact de ces politiques publiques sur l'évolution de différents systèmes agraires en Europe et sur les effets de ces dispositifs, sur la contribution des différents systèmes de production agricoles à la création de biens publics sociaux et environnementaux (Devienne, Garambois et Bazin, 2016). 
(2007), trois à quatre enquêtes technicoéconomiques très approfondies ont été menées pour chacun des treize systèmes de production que nous avons identifiés dans le Jura souabe moyen ( $c f$. annexe), basées sur la collecte de l'ensemble des données permettant d'en caractériser précisément le fonctionnement (ressources, ensemble des éléments de conduite et des performances de chacun des systèmes de culture et d'élevage, données économiques). Ces données ont permis de modéliser un fonctionnement technique type, propre à chacun de ces treize systèmes de production et, sur la base de ce modèle, de mesurer les résultats économiques actuels de chacun d'entre eux (produit brut, consommations intermédiaires, consommations moyennes annuelles de capital fixe, valeur ajoutée nette, subventions, revenu agricole...), pour un système de prix choisi.

\section{Une agriculture familiale tournée de longue date vers la production laitière}

Le plateau du Jura souabe-plus grand ensemble karstique d'Allemagne-est encadré par la vallée très urbanisée du Neckar, qui abrite le couloir industriel Stuttgart-Bodensee. Compris entre 650 et 850 mètres d'altitude, le Jura souabe moyen présente un paysage typique de vallons secs en terres labourables encadrant des interfluves aux sols plus minces occupés par des prairies permanentes et des forêts. Les surfaces agricoles de cette région largement tournée vers l'élevage laitier se composent à parts égales de terres labourables et de prairies permanentes. Le climat montagnard conduit à d'importants reports de stocks fourragers pour alimenter les troupeaux de novembre à mars, mais la concentration des deux tiers des précipitations annuelles (800 à $1000 \mathrm{~mm}$ ) durant une période de croissance végétative resserrée sur sept mois et le fort ensoleillement dont bénéficie la région permettent d'atteindre une production élevée de biomasse par hectare et par an.

Dès la première moitié $\mathrm{du} \mathrm{Xx}^{\mathrm{e}}$ siècle, l'agriculture du Jura souabe se caractérisait par une occupation continue du sol sur les terres labourables, une alimentation des bovins en zéro pâturage (affourragement en vert, betterave et foin) et des reports massifs de stocks fourragers en automne et en hiver qui concouraient à maximiser la production de lait par hectare, au prix d'une intensification poussée en travail. Cette agriculture a connu depuis les années 1950 des gains majeurs de production par hectare et de productivité du travail, accompagnés d'une spécialisation progressive, d'une différenciation poussée des systèmes de production et de la disparition des plus petites exploitations au profit de l'agrandissement des plus vastes.

Nos entretiens historiques montrent que les plus grandes exploitations ont amorcé ce mouvement dès le début des années 1960. La moto-mécanisation, le recours aux engrais de synthèse, la technique d'ensilage (augmentation du nombre de coupes sur les prairies permanentes, introduction facilitée du maïs fourrage à partir des années 1980) permettent ainsi d'accroître la production de fourrages et la densité énergétique des rations. La sélection variétale et le recours accru aux pesticides renforcent la progression des rendements des cultures, facilitent l'introduction du colza dans les rotations dès les années 1970 et le recentrage progressif sur un nombre réduit d'espèces cultivées selon une rotation simplifiée: l'ancienne rotation triennale [céréales d'hiver (blé, orge, épeautre ou seigle)/céréale de printemps (orge ou avoine)/plante sarclée (pomme de terre, betterave) ou légumineuse (trèfle, lentille)] évolue le plus souvent en [colza ou trèfle/blé d'hiver/orge de printemps], puis en [maïs (ou colza)/blé/ orge] à partir des années 1980. L'ensilage de maïs (équilibré de tourteau de soja acheté) n'a eu de cesse dès lors de progresser dans l'assolement et dans l'alimentation 
du troupeau, permettant d'accroître le rendement laitier et la production de lait par hectare au prix de coûteux équipements. Son essor s'accompagne d'une spécialisation des exploitations qui ont pu s'agrandir : élevage laitier pour celles qui ont les moyens d'effectuer de lourds investissements (stabulation libre sur caillebottis, salle de traite) ; élevage porcin sinon.

La réforme de la Politique agricole commune (PAC) de 1992, plus favorable aux céréales et aux oléo-protéagineux, a contribué à renforcer ces évolutions. Le statut marchand et désolidarisé de la terre des quotas laitiers - instauré en Allemagne à partir de 1993 (Barthélémy et Boinon, 2001)-renforce les inégalités cumulatives enregistrées depuis les années 1950. De nombreuses petites exploitations villageoises cessent l'élevage laitier dans les années 1990, en revendant leur quota laitier et en développant de nouveaux systèmes de production moins exigeants en travail et compatibles avec une activité salarié à temps plein (élevage allaitant ou céréaliculture). Grâce au dynamisme fort et ancien dans la région des activités artisanales et de petite industrie (mécanique, machinerie de précision, menuiserie, énergies renouvelables, etc.) (Fel et Miège, 1972 ; Bauer et Schönnamsgruber, 1988), le Jura souabe moyen conserve en effet une forte proportion d'agriculteurs doubleactifs (35 à $80 \%$ de la population agricole selon les communes au début des années 1970 et 65 à $80 \%$ en $2010^{4}$ ) qui va de pair avec une densité élevée de population ( 270 habitants $/ \mathrm{km}^{2}$ en 2014, contre 52 hab/ $\mathrm{km}^{2}$ dans le département français du Jura) et un très faible taux de chômage.

Néanmoins, les plus grandes exploitations laitières équipées alors de salles de traite de $2 \times 6$ postes côtoient toujours, à la fin des années 1990, de petites exploitations villageoises avec traite au pot

4. Statistisches Landesamt Baden-Württemberg. conduites par des retraités. La forte progression de la production de biomasse par hectare enregistrée dans cette région de moyenne montagne et le développement de vastes exploitations laitières à forte disponibilité en capital, contribuent à expliquer le développement rapide, dans le Jura souabe moyen, de la production d'énergie (tout particulièrement de méthane) depuis les années 2000, grâce aux dispositifs incitatifs de la politique énergétique fédérale.

\section{Du solaire au méthane : une progression par étapes de la production d'énergie}

\section{Les soutiens à la production d'énergie solaire du début des années 2000}

Une première loi relative à l'introduction dans le réseau public de courant issu d'énergies renouvelables (Stromeinspeisungsgesetz $z^{5}$ ) est mise en place dès 1991 en Allemagne. Cette loi favorise en premier lieu les installations hydro-électriques et éoliennes. Malgré des prix bonifiés de l'électricité semblables à ceux de l'éolien, les coûts des installations photovoltaïques demeurent élevés et limitent leur développement. En revanche, à partir de 2000, la loi sur les énergies renouvelables (EEG) met fortement l'accent sur l'énergie photovoltaïque. L'électricité d'origine solaire est payée en 2001 au producteur autour de $50 \mathrm{ct} / \mathrm{kWh}$ (en prévoyant par la suite une baisse annuelle de $5 \%$ ), contre 6,2 à $9,1 \mathrm{ct} / \mathrm{kWh}$ pour l'éolien, $7,7 \mathrm{ct} / \mathrm{kWh}$ pour l'hydro-électricité et 8,7 à 10,2 ct/ $\mathrm{kWh}$ pour le courant issu de transformation de la biomasse ${ }^{6}$. De 1999 à 2003, un vaste programme d'installation de panneaux solaires (le 100000 DächerProgramm) est

5. Stromeinspeisungsgesetz : loi sur la fourniture d'électricité.

6. Gesetz für den Vorrang Erneuerbarer Energien (Erneuerbare-Energie-Gesetz-EEG) sowie zur Änderung des Energiewirtschaftsgesetzes und des Mineralölsteuer, vom 29. März 2000. 
mené sous la forme d'octroi de prêts à taux zéro qui permet (fin 2003) de dépasser l'objectif initial national d'une capacité totale de production d'électricité d'origine solaire de 300 mégawatts (MW).

Facilitées par le bon ensoleillement de la région et encouragées par une politique fédérale incitative, les installations photovoltaïques se développent rapidement dès 2000 dans le Jura souabe et constituent aujourd'hui encore la première forme d'énergie produite par l'agriculture souabe. Les panneaux photovoltaïques sont le plus souvent installés sur les toitures à la faveur du renouvellement d'un bâtiment agricole. Nos enquêtes montrent que le rapide retour sur investissement (rentabilisé en moyenne en dix ans), proportionnel à la surface de toiture disponible, incite la plupart des agriculteurs à s'équiper et explique la large gamme de puissance des installations photovoltaïques dans la région (10 à 120 kWh par exploitation). Seuls les éleveurs disposant de très faibles surfaces de bâtiments agricoles (céréaliers double-actifs) et/ou de trop peu de capitaux (petits éleveurs laitiers retraités et éleveurs porcins) n'ont pas les moyens d'accéder à ce revenu complémentaire.

\section{2. Émergence de systèmes de production combinant élevage laitier et unité de méthanisation}

À partir de 2004, la réforme de l'EEG prévoit la réduction des prix bonifiés à l'électricité d'origine éolienne et photovoltaïque et met désormais l'accent sur la production d'éthanol, de diesel et de méthane : les surfaces dédiées aux cultures énergétiques, passées de 500000 ha en 1998 à 900000 ha en 2003 à l'échelle nationale, connaissent une nette accélération et dépassent le 1,9 million d'hectares en 2007, tandis que la construction d'installations de méthanisation connaît une progression extrêmement rapide avec un doublement de la puissance cumulée et du nombre des installations allemandes entre 2003 et 2007 (passée de 750 MWh à 1550 MWh et de 1750 à 3700 unités) (DBFZ, 2011).

Nos enquêtes indiquent que cette orientation de la politique énergétique allemande en faveur de la production de méthane à partir de 2004 amorce une nouvelle étape de différenciation au sein des plus vastes exploitations laitières souabes. Les éleveurs disposant de quotas laitiers élevés grâce à des rachats conséquents maintiennent une stricte spécialisation laitière et poursuivent leurs investissements dans le renouvellement et l'accroissement de la capacité des bâtiments et équipements (salle de traite de $2 \times 8$ postes pour des troupeaux dépassant les 100 vaches laitières). Les autres, moins bien dotés en quotas laitiers, se tournent dès 2004 vers une double spécialisation en privilégiant l'installation d'une unité de méthanisation de 75 à $200 \mathrm{kWh}$ plutôt que l'achat de quotas laitiers supplémentaires. Ils maintiennent des troupeaux de 40 à 60 vaches laitières sans nouvel investissement dans les bâtiments d'élevage ou l'équipement de traite.

\section{Capacité croissante des méthaniseurs et premières spécialisations dans la production d'énergie}

Les réformes de 2008-2009 de l'EEG marquent un net renforcement des soutiens aux producteurs de méthane issu de la fermentation anaérobie d'un substrat biologique et prend plus finement en compte ce processus et la coproduction de chaleur. De nombreux composants organiques (effluents (lisier, fumier), déchets verts, paille, herbe...) peuvent entrer dans la composition de ce substrat, mais aussi des cultures plus spécifiquement énergétiques telles que le maiis fourrage ou les céréales ensilées. L'énergie contenue dans le méthane produit est ensuite convertie pour $38 \%$ en électricité et pour $47 \%$ en chaleur. Cette chaleur peut être directement utilisée afin de maintenir la température dans le digesteur ; le reste est mobilisé à proximité quand cela est possible (chauffage 
Tableau 1. Prix de base et bonus versés aux producteurs allemands d'électricité dérivée de méthane entre 2009 et 2012 (en cents d'euros par kWh produit)

\begin{tabular}{lccc}
\hline & \multicolumn{3}{c}{ Capacité de l'installation } \\
\cline { 2 - 4 } & jusqu'à $150 \mathrm{kWh}$ & jusqu'à 500 kWh & jusqu'à 5 MWh \\
\hline Soutien de base & 11,7 & 9,2 & 8,3 \\
Bonus « Nawaro " & 7 & 7 & 4 \\
Bonus Lisier & 4 & 1 & 0 \\
Bonus Couplage thermique & 3 & 3 & 3 \\
Bonus Technologique & jusqu'à 2 & jusqu'à 2 & jusqu'à 2 \\
\hline
\end{tabular}

Source : Gesetz zur Neuregelung des Rechts der Erneuerbaren Energien im Strombereich und zur Änderung damit zusammenhängender Vorschriften, vom 25, Oktober 2008.

collectif), sinon perdue vers l'atmosphère. Cette réforme prévoit pour les producteurs d'électricité dérivée du méthane un prix de base du KWh garanti pour vingt ans auquel s'ajoutent différents bonus, qui décroissent par tranche à mesure qu' augmente la capacité de l'installation (tableau 1) :

- le bonus NaWaRo (Nachwachsende Rohstoff-Bonus) est perçu si l'alimentation du méthaniseur ne repose que sur des produits ou sous-produits végétaux qui n'auraient «pas d'autres usages possibles »;

- le bonus lisier (Gülle-Bonus) est alloué si le substrat comporte en masse au moins $30 \%$ de lisier ;

- le bonus couplage thermique (KraftWärme-Kopplung-Bonus) est octroyé aux installations qui valorisent le surplus d'énergie thermique produite ;

- le bonus technologique (Technologiebonus) bénéficie aux installations qui présentent une plus grande efficience énergétique.

Ces réformes s'accompagnent d'un renforcement de la puissance cumulée et du nombre des unités de méthanisation allemandes, qui progressent de 1550 à $2450 \mathrm{MWh}$ et de 3700 à 5900 installations entre 2007 et 2010 (DBFZ, 2011). Les agriculteurs détiennent alors plus de $80 \%$ des installations de moins de $500 \mathrm{kWh}$. En 2012, les 2437000 ha destinés aux cultures énergétiques représentaient près de $15 \%$ de la Surface Agricole Utile (SAU) allemande et cette même année les surfaces destinées à la production de méthane atteignaient 1158000 ha, dépassant celles dédiées à la production de diesel (786 000 ha $)^{7}$. Parallèlement, les prix garantis de l'énergie photovoltaïque diminuent progressivement chaque année.

Notre recherche illustre le fait que l'agriculture souabe s'inscrit pleinement dans ces tendances. Si les installations photovoltaïques les plus anciennes bénéficient toujours d'un prix élevé de l'électricité, la forte baisse des prix bonifiés depuis quelques années n'encourage plus les agriculteurs à étendre ces installations. La capacité des unités de méthanisation connaît en revanche une nette progression depuis 2008. Les exploitations associant élevage laitier et méthaniseur sont désormais équipées d'installations de 150 à $200 \mathrm{kWh}$ et sont généralement implantées suffisamment à proximité des villages pour disposer d'un système couplé permettant de valoriser une partie de la chaleur dégagée lors du processus de fermentation en chauffant habitations ou bâtiments publics, et de bénéficier ainsi d'une prime. La majorité des élevages bovins étant conduits toute l'année dans des bâtiments sur

7. D'après Fachagentur Nachwachsende Rohstoffe (FNR). 


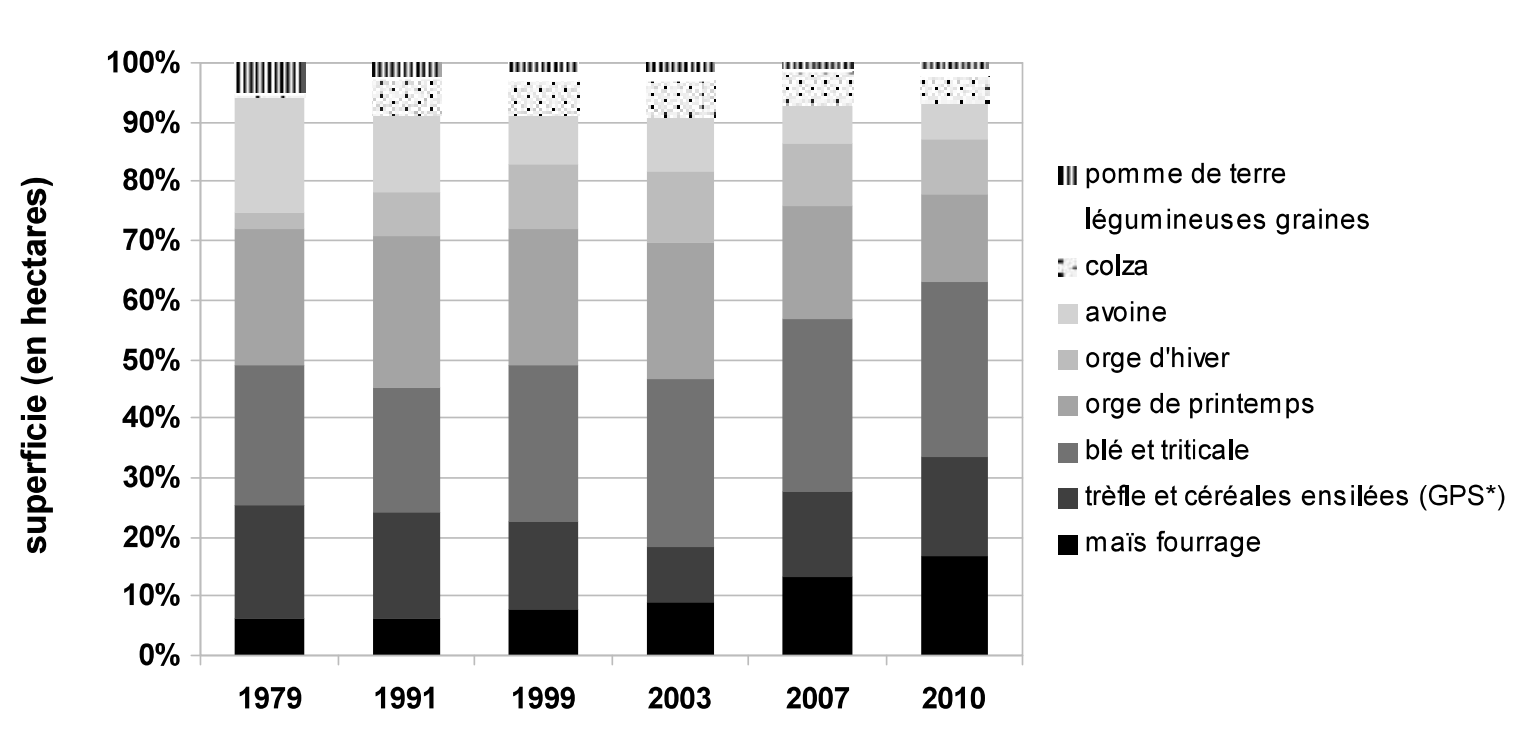

Source : Statistisches Landesamt Baden-Württemberg/ ${ }^{*}$ GPS : Ganzpflanzensilage.

caillebottis, la plupart des producteurs de méthane sont en mesure d'intégrer plus de $30 \%$ en masse de lisier dans le substrat qui leur permet de bénéficier du bonus associé.

Les agriculteurs souabes associant production de lait et de méthane les mieux dotés en terres labourables peuvent introduire plus largement dans leur assolement maïs fourrage et mélanges céréaliers à base de triticale et de seigle récoltés en vert, substrats particulièrement énergétiques (un demi-hectare de maïs fourrage fournit autant-un $\mathrm{kW}$ annuel-qu'un hectare de prairie permanente conduit avec trois coupes d'ensilage). À la faveur du perfectionnement technologique, et encouragés par un prix de l'électricité garanti sur vingt ans, ils ont rapidement effectué un nouveau saut d'investissement en abandonnant la production laitière et en s'équipant d'un second moteur afin de doubler la puissance de leur unité de méthanisation $(250$ à $300 \mathrm{kWh})$, voire en construisant un second méthaniseur portant leur capacité de production jusqu'à $500 \mathrm{kWh}$. Des agriculteurs à la tête de vastes troupeaux les ont rapidement rejoints, abandonnant l'élevage laitier pour basculer directement vers une stricte spécialisation de production de méthane. La puissance moyenne des 893 installations de méthanisation du Bade-Wurtemberg atteignait ainsi $357 \mathrm{kWh}$ en moyenne en $2014^{8}$.

\section{Bouleversement des systèmes de production sous l'essor des unités de méthanisation}

\section{Transformations des assolements, des systèmes de culture et des échanges entre exploitations}

L'évolution des besoins liés au développement rapide des méthaniseurs dans la région s'accompagne dès 2004 de la progression du maïs fourrage et des céréales récoltées en vert (Ganzpflanzensilage [GPS]) dans l'assolement de la région, passés de moins de $20 \%$ sur les terres labourables en 2003 à plus du tiers en 2010, au détriment des céréales secondaires et du colza (figure 1).

8. https://www.foederal-erneuerbar.de/landesinfo/bundesland/BW/kategorie/bioenergie/ auswahl/189-anzahl_und_dichte_vo/\#goto_189. 


\section{FAITS ET CHIFFRES}

D'après nos enquêtes, la rotation [maïs ou colza// blé, triticale ou orge d'hiver // orge de printemps ou avoine], encore largement pratiquée dans le Jura souabe, évolue en effet au sein des exploitations combinant production de lait et de méthane vers une rotation triennale [maiis // triticale ou seigle en vert/maïs // orge d'hiver] où un tiers de la sole (cultures de seconde année) est directement dédié à la production de méthane. Les exploitations spécialisées dans la production de méthane ont même cessé de produire des céréales grains en pratiquant désormais une rotation biennale [maïs/seigle ou triticale en vert // maïs/raygrass d'Italie et trèfle incarnat] où l'occupation continue du sol permet de maximiser la production de biomasse à l'hectare, intégralement conservée sous forme ensilée et dédiée à la stricte production de méthane.

Outre les superficies directement exploitées, les producteurs d'énergie complètent leurs productions végétales d'achats de maïs fourrage, de céréales récoltées en vert et même d'herbe sur pied. Ces achats étendent les bouleversements apportés à leurs propres systèmes de culture à ceux mis en œuvre au sein des exploitations fournisseuses, à savoir les céréaliculteurs double-actifs, qui dans leurs rotations remplacent de plus en plus fréquemment le colza par du maïs fourrage. Les fourrages alimentant les méthaniseurs portent également sur ceux récoltés pour la vente par les céréaliculteurs double-actif sur leurs terres demeurées en prairies permanentes. Si la première coupe est encore récoltée en foin et destinée aux haras locaux, la seconde coupe de foin, auparavant vendue aux éleveurs laitiers de la région voisine de l'Allgaü, est désormais vendue aux producteurs de méthane sous forme d'ensilage.

La gestion des effluents des exploitations d'élevage est également affectée par l'essor des méthaniseurs. Afin de bénéficier du bonus lisier concédé pour les
150 premiers $\mathrm{kWh}$ produits lorsque la part de cet effluent dans le substrat dépasse en masse les $30 \%$, les agriculteurs spécialisés dans la production de méthane qui ne disposent plus de lisier en propre, établissent des contrats avec un ou plusieurs de leurs voisins éleveurs laitiers. Moyennant la prise en charge du transport de l'ensemble de ces effluents, cela leur permet d'alimenter leur installation avec le lisier et de restituer aux éleveurs l'azote organique qu'il contient sous une forme minérale, résidu du digestat appauvri en carbone.

Au-delà de l'évolution de l'assolement, le développement rapide de la production de méthane dans la région et les considérables besoins en biomasse des producteurs d'énergie contribuent ainsi à bouleverser en profondeur le fonctionnement $\mathrm{du}$ système agraire souabe et conduit au détournement d'une part croissante de la matière organique (fourrages, céréales plante entière, fumier et lisier) vers la production de méthane, au détriment des restitutions de carbone aux sols, de la reconstitution de leur taux d'humus et du maintien de leur stabilité structurale. Ces évolutions contribuent également à simplifier les rotations pratiquées au profit du maïs et à recourir davantage à la fertilisation chimique et aux pesticides, dont l'usage accru est rendu artificiellement rentable par les hauts niveaux de prix garantis pour l'électricité (et indirectement des fourrages vendus).

\section{Renforcement des écarts de revenus et recul de la richesse revenant au travail agricole}

Nos travaux montrent que les exploitants à la tête des systèmes producteurs de méthane enregistrent les plus hauts revenus agricoles du Jura souabe. La production de méthane au sein d'unités de capacité dépassant les $350 \mathrm{kWh}$ mobilise par ailleurs des surfaces considérables pour la région (125 à 150 ha par actif familial), intégralement dédiées à la production de fourrages 
Figure 2. Distribution de la valeur ajoutée nette entre revenu agricole familial avant subventions, salaires, rente foncière et intérêt du capital, pour différents systèmes de production souabes (avec chacun deux temps pleins familiaux et un mi-temps salarié)

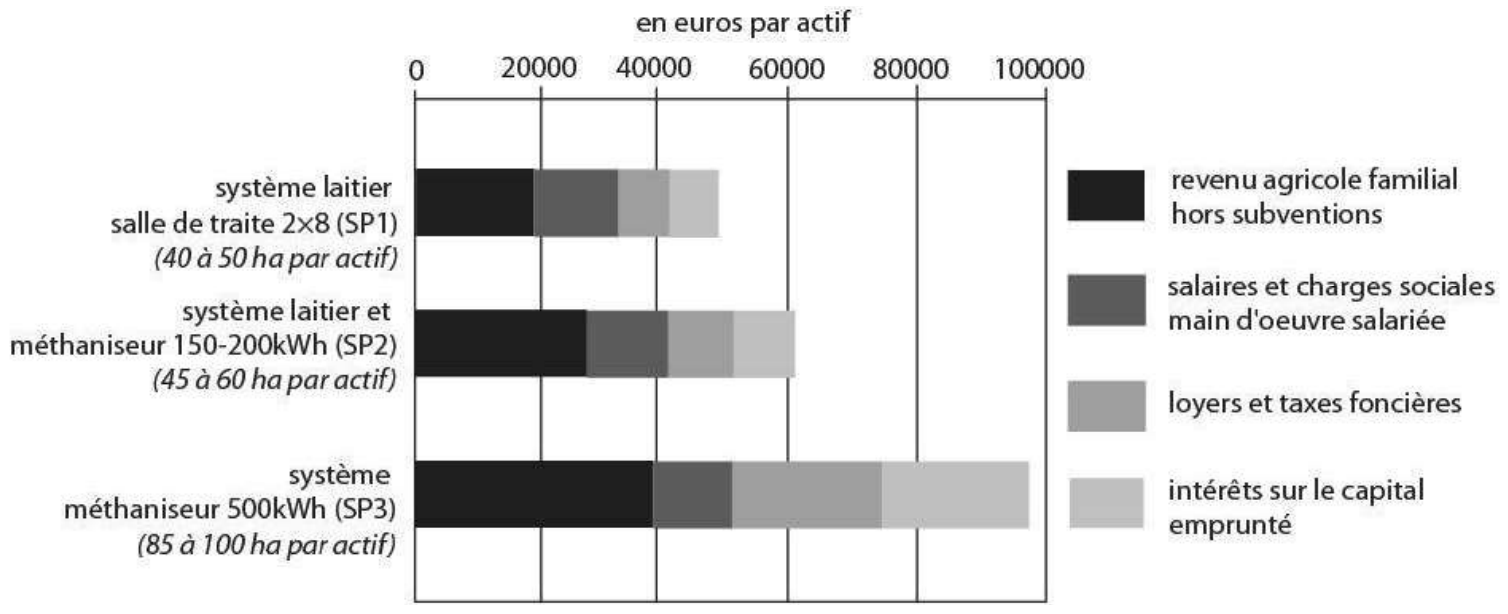

Source : enquêtes, système de prix 2011-2012.

récoltées, l'ensilage créant des pointes de travail que la seule main-d'œuvre familiale n'est pas en mesure de prendre en charge. Ces agriculteurs emploient ainsi durant toute la période végétative (mai à octobre) un salarié issu des pays de l'Est (Pologne, Roumanie) ou de Russie, démarche facilitée par la souplesse de la législation du travail migratoire outre-Rhin puisque les travailleurs polonais et roumains n'ont désormais plus besoin de permis de travail pour exercer en Allemagne.

Dans le Jura souabe où le taux de chômage extrêmement bas avoisine les 3,5\%, nos entretiens indiquent que le recours à une main-d'œuvre étrangère deux fois moins coûteuse par heure travaillée, n'est pas propre aux systèmes spécialisés dans la production de méthane: lorsque les parents retraités deviennent trop âgés pour participer à la traite, les exploitations à deux actifs dont le troupeau dépasse les 100 vaches privilégient pour l'instant l'embauche à l'année d'un salarié d'Europe de l'Est plutôt que l'investissement dans un équipement de traite de plus grande capacité. En revanche, nos résultats permettent également de montrer que la captation de la richesse créée par la maind'œuvre salariée est plus forte au sein des systèmes producteurs de méthane au regard des systèmes laitiers spécialisés : $25 \%$ de la valeur ajoutée nette ${ }^{9}$ agricole créée par l'ensemble des actifs au sein des vastes exploitations laitières spécialisées équipées d'une salle de traite $2 \times 8$ postes (SP1) revient à la rémunération des salariés, contre $20 \%$ en système associant élevage laitier et méthaniseur (SP2) et seulement $12 \%$ en système spécialisé dans la production de méthane (SP3) (figure 2).

Ainsi, les prix élevés de l'électricité versés aux producteurs permettent avant tout à la main-d'œuvre familiale de couvrir des intérêts du capital empruntés, des frais de fermage et une taxe foncière par actif familial bien supérieurs, tout en maintenant la rémunération de leur travail à hauteur d'environ $40 \%$ de la valeur ajoutée créée par l'ensemble des actifs travaillant sur

9. Valeur ajoutée nette $=$ Produit brut (valeur de la production agricole, hors aides) - Consommations intermédiaires - Consommations annuelles moyennes de capital fixe (dépréciation annuelle des équipements et bâtiments). 
l'exploitation (ratio commun aux trois systèmes présentés en figure 2). Chaque actif familial perçoit ainsi un revenu agricole deux fois supérieur en mettant en œuvre un système avec méthaniseur de $500 \mathrm{kWh}$ au regard d'un système strictement laitier à salle de traite $2 \times 8$ postes. Tout en offrant de très hauts niveaux de revenu à certains des agriculteurs familiaux souabes, l'essor de la production de méthane dans le Jura souabe conduit ainsi au développement de systèmes qui rémunèrent de façon croissante le capital et la rente foncière, respectivement $35 \%$ en système laitier (SP1), $40 \%$ en système associant productions laitière et de méthane (SP2) et près de $50 \%$ en système strictement producteur de méthane (SP3), et ce au détriment du travail salarié.

\section{Flambée récente des prix du fermage et insécurisation de l'accès au foncier}

Un tiers des terres agricoles du Jura souabe moyen est aujourd'hui exploité en fairevaloir direct et deux tiers en fermage. Fort de son dynamisme économique, le Jura souabe a connu depuis une trentaine d'années une accélération du rythme de construction de lotissements. Les terres agricoles à proximité des villages, souvent labourables, n'en sont que plus convoitées : peu de terres sont ainsi offertes à la vente et leur loyer n'a eu de cesse de progresser. Ce phénomène se renforce à mesure que les agriculteurs à temps plein tentent d'agrandir leurs exploitations, dans un contexte où nombre d'enfants d'agriculteurs continuent à exploiter les terres labourables de l'exploitation familiale en parallèle d'une activité salariée.

Dans ce contexte, le haut niveau des prix garantis pour l'électricité permet aux producteurs de méthane de louer des terres à un prix supérieur, tandis que le développement récent d'unités de méthanisation de grande capacité crée des besoins en fourrages qui dépassent très largement les seuls volumes récoltés sur les terres jusque-là exploitées par ces agriculteurs. Sous l'effet de cette considérable progression de la demande, les prix du fermage flambent depuis quelques années et ont doublé en monnaie constante entre la période 2005-2010 et l'année 2012, passant de 150 à 300 euros par hectare de prairie permanente et de 200 à 400 voire 500 euros l'hectare pour les terres labourables (source : enquêtes).

Favorisés par l'absence d'encadrement des prix du fermage et de la durée du bail en Allemagne (Jaubertie, 2011), les contrats de fermage qui duraient auparavant dix ans doivent désormais être reconduits toutes les années pour les terres labourables et sont saisis par les mieux offrants. La forte progression des prix du fermage fragilise les exploitations à temps plein, notamment les exploitations porcines dont les revenus se dégradent avec l'évolution défavorable des prix, et qui n'ont pas les moyens de rivaliser avec les exploitations productrices de méthane dans l'accès au foncier. Les céréaliculteurs double-actifs, propriétaires des terres qu'ils exploitent, ne sont pas directement affectés par la flambée des prix du fermage, mais en dessous de 20 ha et si le prix des grains baisse, ils estiment plus rentable de louer leurs terres aux producteurs de méthane plutôt que de produire des grains destinés à l'alimentation, contribuant au recul conjoncturel des productions agricoles au profit du méthane.

\section{Des politiques agricoles et rurales insuffisantes pour contrebalancer la politique énergétique fédérale}

La régionalisation mise en place en Allemagne dans le cadre du découplage des aides du premier pilier de la PAC s'est traduite dans le Jura souabe par un soutien de base assez homogène entre cultures annuelles et prairies permanentes 
(respectivement 309 et 270 euros par hectare) pour le calcul des Droits à paiement unique (DPU), référence pour le calcul des Droits à paiement de base (DPB) depuis 2015. Sans remettre en cause des soutiens du premier pilier proportionnels à la surface, très favorables aux producteurs de méthane qui disposent des plus vastes surfaces par actif, elle a permis un rééquilibrage des soutiens en faveur des unités de production utilisant une part importante de prairies permanentes. En l'absence de mesures spécifiques de conditionnalité, elles n'influent en revanche pas sur le mode de conduite de ces prairies, en particulier le niveau de fertilisation azotée.

Couvrant $40 \%$ des aides au développement agricole accordées par le BadeWurtemberg, le programme MEKA lancé en 1992 (second pilier de la PAC), renforcé depuis 2001 de soutiens fédéraux ciblés, contribue depuis quinze ans au développement de l'agriculture biologique dans le Bade-Wurtemberg, Land qui représente aujourd'hui à lui seul $30 \%$ des exploitations allemandes inscrites en agriculture biologique et assure avec la Bavière la production de $90 \%$ du lait bio collecté en Allemagne (Institut de l'Élevage, 2012). Nos enquêtes montrent que les systèmes de production de la région inscrits en agriculture biologique et basés sur un fonctionnement plus économe contribuent plus largement au maintien de l'emploi agricole et à la préservation de l'environnement : en élevage bovin laitier grâce à la reprise de l'affourragement en vert (prairies à base de légumineuses) au détriment du maïs fourrage, voire au recours au pâturage abandonné de longue date dans la région; en grandes cultures, grâce à la diversification des cultures, à l'allongement des rotations, à une place accrue des légumineuses et à une association plus étroite à l'élevage.

Le Jura souabe a par ailleurs bénéficié dès 1993 des premiers programmes Plan pour le maintien et le développement de la nature et de l'environnement (PLENUM) lancés par le ministère de l'Environnement et conduits par les Länder, qui ont porté sur le lancement de marques de produits régionaux élaborés à partir de céréales et légumineuses graines cultivées dans la région et sur des soutiens ciblés à l'investissement (développement de meuneries locales, de magasins de producteurs et de l'accueil à la ferme). Ces initiatives contribuent à la diversification de l'assolement et à des itinéraires techniques moins gourmands en intrants pour les agriculteurs engagés, grâce à la réintroduction dans les rotations d'espèces plus résistantes et/ou fournisseuses d'azote (épeautre, lentilles, etc.). Si les programmes régionaux concernent un grand nombre d'unités de production, les surfaces agricoles concernées demeurent limitées et les mesures segmentées ne portent pas sur un soutien à des systèmes de production spécifiques, dont le fonctionnement global ferait la preuve d'une plus grande contribution au maintien de l'emploi et à la préservation de l'environnement. Avec 1,7 million d'euros d'aides sur la période 2001-2007 pour le programme PLENUM, les moyens financiers mis en œuvre par le Bade-Wurtemberg en matière de développement agricole ne peuvent de toute façon pas rivaliser avec la politique énergétique fédérale, ni permettre de bâtir un dispositif suffisamment incitatif pour espérer contrebalancer les effets de l'essor de la production de méthane dans la région.

$$
\begin{gathered}
* \\
* \quad *
\end{gathered}
$$

Centrée à ses débuts sur le soutien aux installations photovoltaïques et éoliennes, et réorientée depuis 2004 vers les soutiens à la production de diesel, d'éthanol et de méthane, la politique énergétique fédérale allemande a conduit certaines des plus vastes exploitations laitières du Jura souabe à intégrer un méthaniseur à leur système de production bovin laitier, voire à se spécialiser 


\section{FAITS ET CHIFFRES}

dans la seule production d'énergie. Le développement récent de ces unités de méthanisation dans la région se solde par de nombreux effets sociaux et environnementaux négatifs. La production de méthane s'effectue en effet au détriment des productions agricoles dédiées directement ou indirectement à l' alimentation humaine. Elle s'avère également discriminante en raison des niveaux d'investissement particulièrement élevés qu'elle requiert et source d'un renforcement des inégalités de rémunération $\mathrm{du}$ travail agricole et d'accès au foncier. Elle est délétère au plan environnemental pour la région (appauvrissement du taux d'humus des sols, simplification des rotations, usages accrus d'intrants).

Ces effets, à rebours du développement d'une agriculture plus durable (sécurité alimentaire, emploi, préservation de l'environnement), constituent un véritable paradoxe pour un dispositif visant à la souveraineté énergétique grâce à des énergies dites « renouvelables ». Avec des prix stables et élevés de l'électricité concédés aux unités de méthanisation montées entre 2009-2012 et garantis sur vingt ans, les producteurs d'énergie du Jura souabe sont, en outre, placés dans des conditions de production et des perspectives bien plus sécurisantes que ne l'offrent la PAC et les politiques publiques régionales aux éleveurs laitiers soumis aux incertitudes liées à l'abandon du régime européen de quotas laitiers et tributaires d'un prix du lait accusant baisse tendancielle et volatilité croissante.
Face au prix élevé de l'électricité pour le citoyen allemand et au rythme jugé trop rapide de conversion des terres agricoles à des fins de production d'énergie, de nombreuses critiques se sont élevées en Allemagne depuis 2012 (notamment suite au bilan dressé dans une évaluation de l'Académie des Sciences allemande Leopoldina (Nat. Akad. Wissen. Leopoldina, 2012)) obligeant le gouvernement allemand à marquer, avec la réforme de 2014 de l'EEG, un net infléchissement dans sa politique énergétique (DBFZ, 2014). Sans s'appliquer aux installations construites avant août 2014, ces mesures prévoient l'abaissement drastique de l'accroissement annuel d'électricité produite à partir de biomasse et un prix garanti au producteur ramené à 13,7 cts $€$ par $\mathrm{kWh}$ pour les installations de puissance inférieure à $150 \mathrm{kWh}$ et à $11,8 \mathrm{cts} €$ pour celles de 150 à $500 \mathrm{kWh}^{10}$, et semblent s'être déjà traduites par une stagnation de la capacité de production d'électricité à partir de biomasse en Allemagne entre 2014 et 2015 (BDEW, 2016). Combinée à la dernière réforme de la PAC (notamment la revalorisation, en Allemagne, des aides du premier pilier pour les 46 premiers hectares à partir de 2015) et au développement de systèmes plus économes, souvent inscrits en agriculture biologique (prix du lait supérieur et plus stable), contribuant plus largement au développement durable, cette mesure pourrait contribuer à ouvrir une autre voie pour l'agriculture souabe. 


\section{RÉFÉRENCES BLIOGRAPHIQUES}

Barthélémy D., Boinon J.-P. (2001). La gestion des quotas laitiers dans les 3 pays membres de l'Union européenne : objectifs marchands versus objectifs non marchands. Journée du département ESR : Quelle politique laitière pour l'Union européenne, INRA, 8 octobre 2001.

Bauer E.W., Schönnamsgruber H. (1988). Das große Buch der Schwäbischen Alb, Stuttgart, Theiss Verlag, $216 \mathrm{p}$.

BDEW (Bundesverband der Energie- und Wasserwirtschaft e. V) (2013). Erneuerbare Energien und das EEG: Zahlen, Fakten, Grafiken (2013), Berlin, BDEW.

BDEW (Bundesverband der Energie- und Wasserwirtschaft e. V) (2016). Erneuerbare Energien und das EEG: Zahlen, Fakten, Grafiken (2016), Berlin, BDEW.

DBFZ (Deutsches BiomasseForschungs Zentrum) (2011). Nachhaltige Biogas erzeugung in Deutschland-Bewertung des Wirkungen des EEG, Berlin, DBFZ.

DBFZ (Deutsches BiomasseForschungs Zentrum)(2014). Vorbereitung undBegleitung der Erstellung des Erfahrungsberichts 2014 gemäss §65 EEG-Wissenschaftlicher Bericht, Berlin, DBFZ.

Cochet H., Devienne S. (2006). Fonctionnement et performances économiques des systèmes de production agricole: une démarche à l'échelle régionale. Cahiers Agriculture, vol. $15, \mathrm{n}^{\circ} 6$, pp. 578-583.

Cochet H., Devienne S., Dufumier M. (2007). L'agriculture comparée: une discipline de synthèse ? Économie rurale, $\mathrm{n}^{\circ} 297-298$, pp. 99-112.

Deshaies M. (2014). Ambiguïtés et limites de la transition énergétique en Allemagne. VertigO, la revue électronique en sciences de l'environnement, vol. 14, $\mathrm{n}^{\circ} 3$, http:// vertigo.revues.org/15515 ; DOI:10.4000/ vertigo. 15515.

Devienne S., Garambois N., Bazin G. (2016). Systèmes de production agricoles et production de biens publics: les enseignements d'une approche en termes de système agraire. In Berriet-Solliec M. (dir.), La production de biens publics en agriculture. Quelle prise en compte par la PAC? Dijon, Educagri Éditions, pp. 79-109.

Fel A., Miège J. (1972). Transformation et urbanisation des campagnes en Allemagne fédérale, Annales de géographie, $\mathrm{n}^{\circ} 447$, pp. 579-593.

Institut de l'Élevage (2012). Les Allemagnes laitières. Dossier économie de l'élevage, $\mathrm{n}^{\circ} 426$.

Jaubertie C. (2011). Histoire et politique foncière de l'Allemagne. Études Agter, ${ }^{\circ} 11$.

Mautz R., Byzio A., Rosenbaum W. (2008). Auf dem Weg zur Energiewende. Die Entwicklung der Stromproduktion aus erneuerbaren Energien in Deutschland. Göttingen, Universitätsverlag Göttingen.

Nationale Akademie der Wissenschaften Leopoldina (2012). Bioenergie-Möglichkeiten und Grenzen. Halle (Saale), 56 p.

Reboul C. (1976). Mode de production et systèmes de culture et d'élevage. Économie rurale, $\mathrm{n}^{\circ} 112$, pp. 55-65.

Thoroe C. (2002). Répartition des compétences de politique agricole entre Länder, État fédéral et Union européenne. Économie rurale, $\mathrm{n}^{\circ}$ 268-269, pp. 68-77.

Vue S. (2012). Entre tradition laitière et "énergie-culture». Analyse-diagnostic d'une petite région agricole de l'Alb Souabe moyen. Mémoire de Master recherche AgroParisTech/Paris X, Paris. 


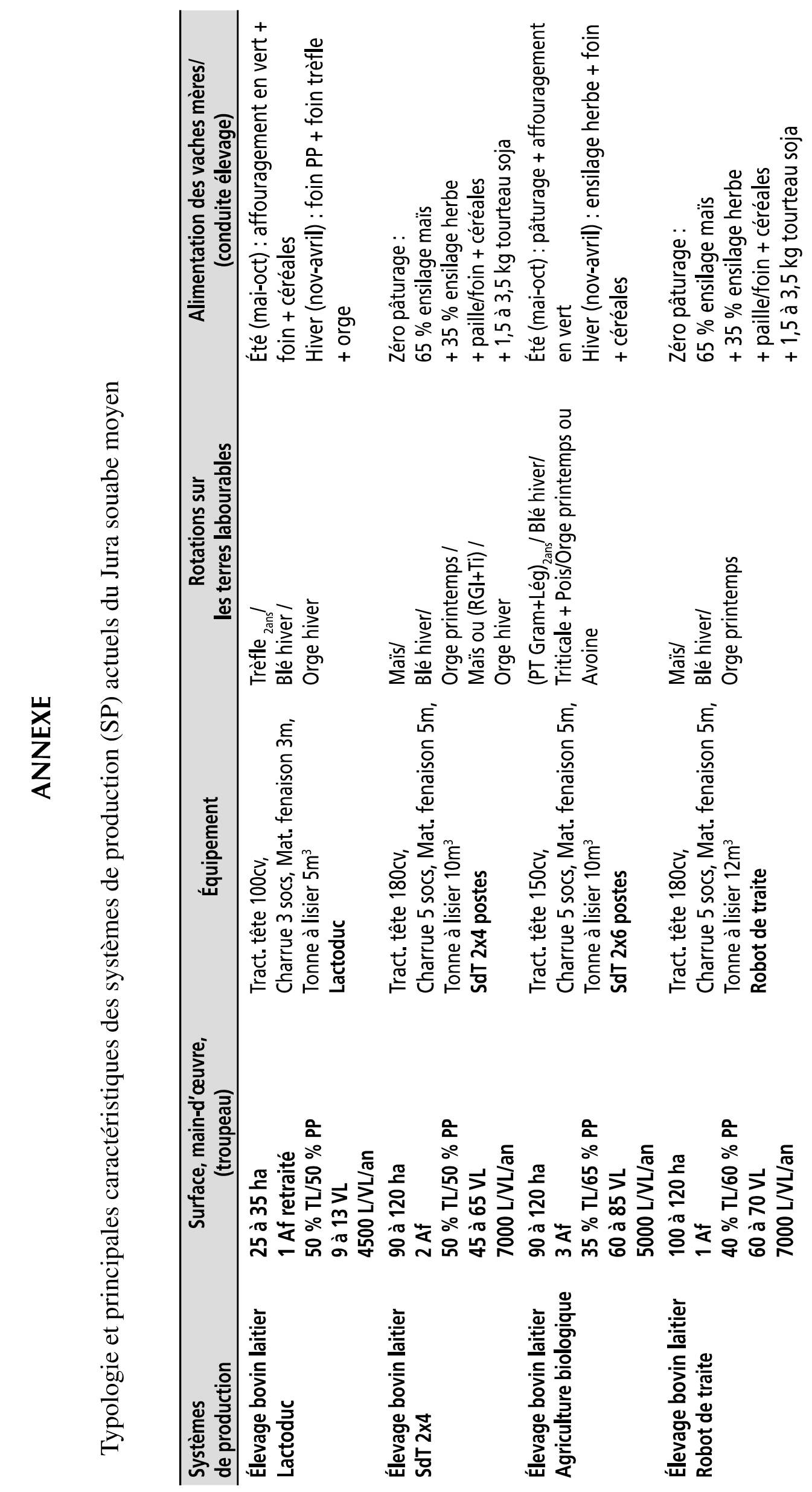



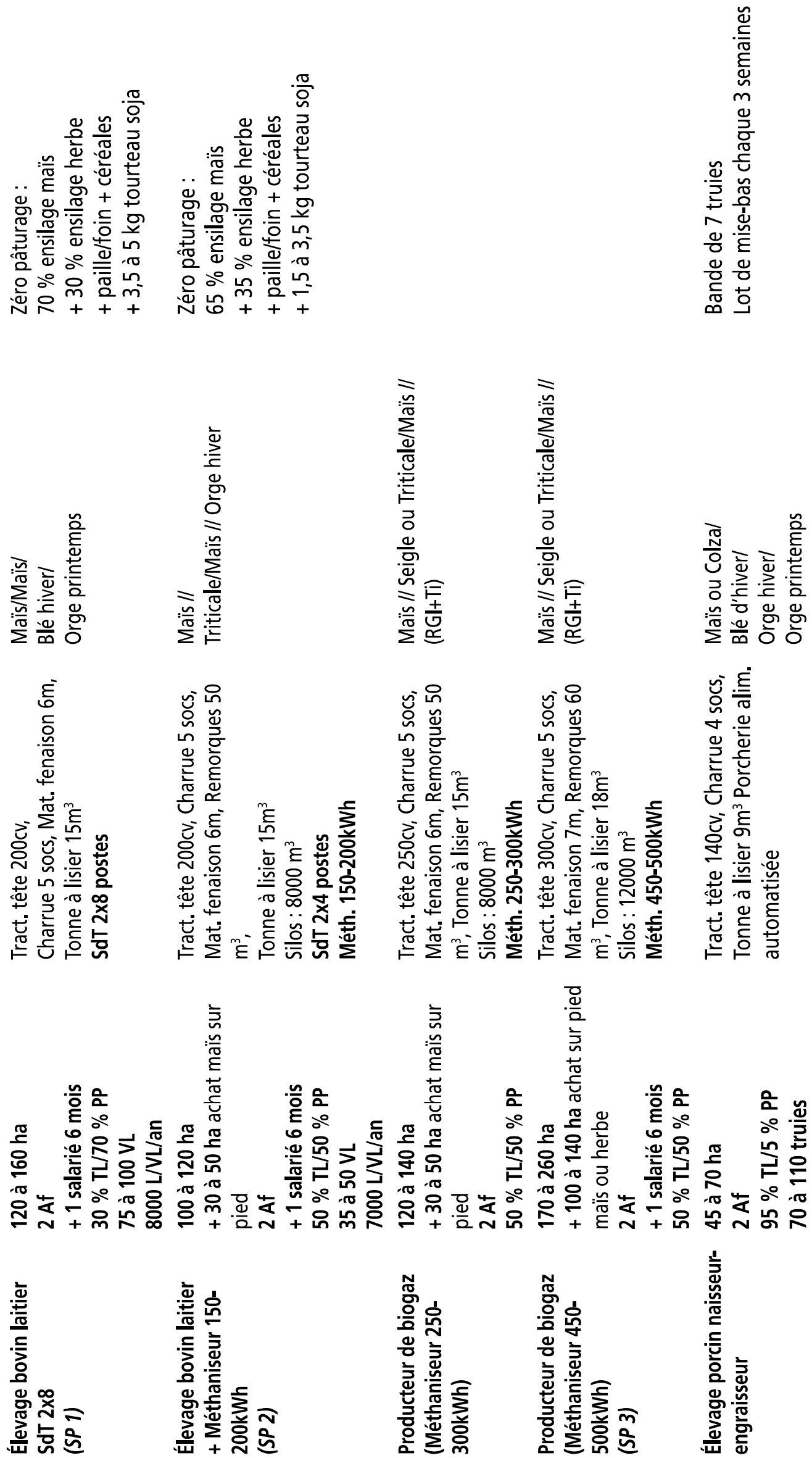

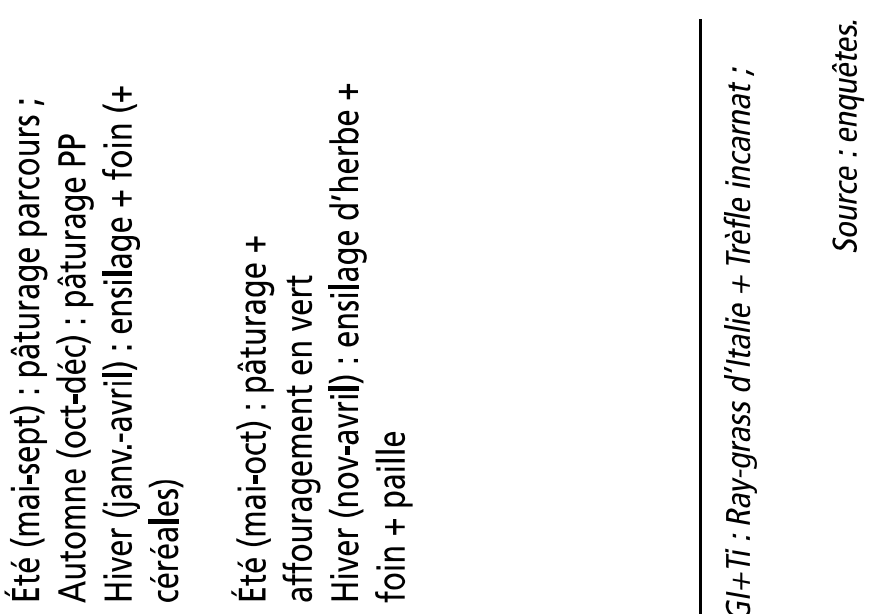

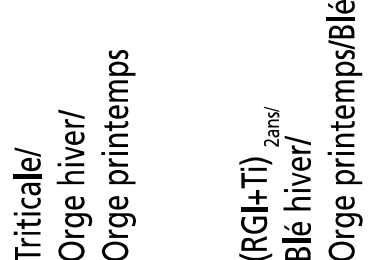

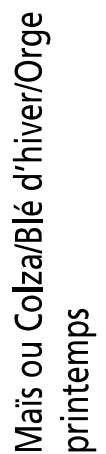

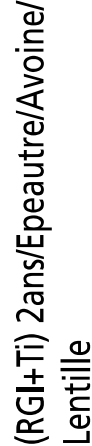

$\frac{.0}{\frac{0}{\pi}}$

迄

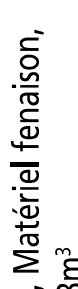

¿ $\sum^{\pi} \sum_{\infty}^{\infty}$ 음

范

売守

壱

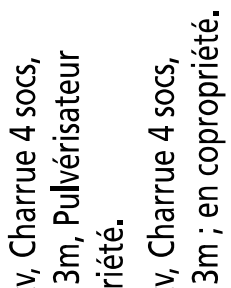

苍

웅 흥

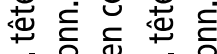

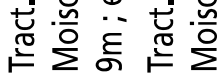

뉼 苋

은

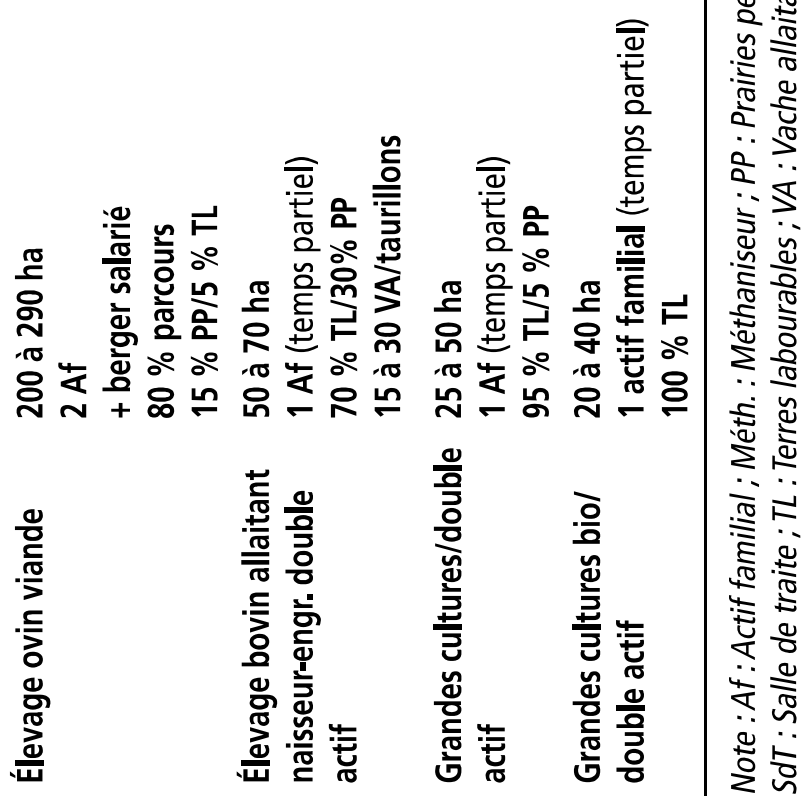

